\title{
Transverse shift of helical beams and sub-diffraction imaging
}

\author{
Thomas Brunet and Jean-Louis Thomas* \\ Institut des NanoSciences de Paris, Université Pierre et Marie Curie Paris 06, \\ CNRS UMR 7588, 140 rue de Lourmel, 75015 Paris, France \\ Régis Marchiano \\ Institut Jean Le Rond D'Alembert, Université Pierre et Marie Curie Paris 06, \\ CNRS UMR 7190, 4 place Jussieu, 75005 Paris, France
}

(Dated: June 17, 2010)

\begin{abstract}
An imaging technique is here proposed to overcome the classical 'diffraction limit' by using helical beams. This technique and the analysis presented are valid for all kinds of waves (either optical or acoustical) as long as the field can be considered as scalar. We show that the stable structure of such phase singularities turns out to be appropriate to measure both the position and the diameter of sub-diffraction circular apertures. The property used is a shift of the scattered vortex. Its location is obtained with a very high resolution thanks to a non-classical correlation method exploiting the super-oscillating property of a vortex near its axis. This theoretical analysis is supported by acoustic experiments performed underwater evidencing sub-diffraction imaging.
\end{abstract}

According to the Abbe-Rayleigh's criterion, it is well-known that conventional imaging systems with a focal length $F$ and a diameter aperture $D$ can not achieve a resolution beyond the diffraction limit $(\sim \lambda F / D)$ at the wavelength $\lambda$. Such a 'physical barrier' not only represents the minimum distance between two distinguishable adjacent objects but also the size of the smallest object that the system can resolve. However, many approaches proved useful to overcome the diffraction limit such as hyperlenses recently developed in optics [1] as well as in acoustics [2]. As noted by Zheludev [3]: "not only are superlenses a promising choice: concepts such as super-oscillations could provide feasible alternative". Super-oscillations have been proposed by Berry and Popescu [4]. Helical waves possess this property. Close to their axis, the field amplitude decreases towards zero and the phase becomes singular [5]. These screw-type dislocations [6], studied in singular optics [7], are also known as optical vortices due to the angular momentum they carry [8] providing optical spanners [9]. Similarly, the acoustical vortices [10] carry a pseudo angular momentum [11] that can be transferred to matter setting objects in rotation [12].

In a transverse plane, the number of jumps of $2 \pi$ achieved by the phase on a close contour is called the topological charge $l$. The classical features of such structures are depicted in Figs. 1(a,b) showing the amplitude RMS and phase for an experimental vortex of topological charge $l=1$. Whatever the radial distance $r$ from the singularity is, the phase increment for a complete turn is constant and equal to $2 \pi l$ as shown in Fig. 1(b). Consequently, it oscillates increasingly fast by getting closer to the vortex core without any apparent physical limits due to evanescent modes suggesting super-oscillation phenomenon.
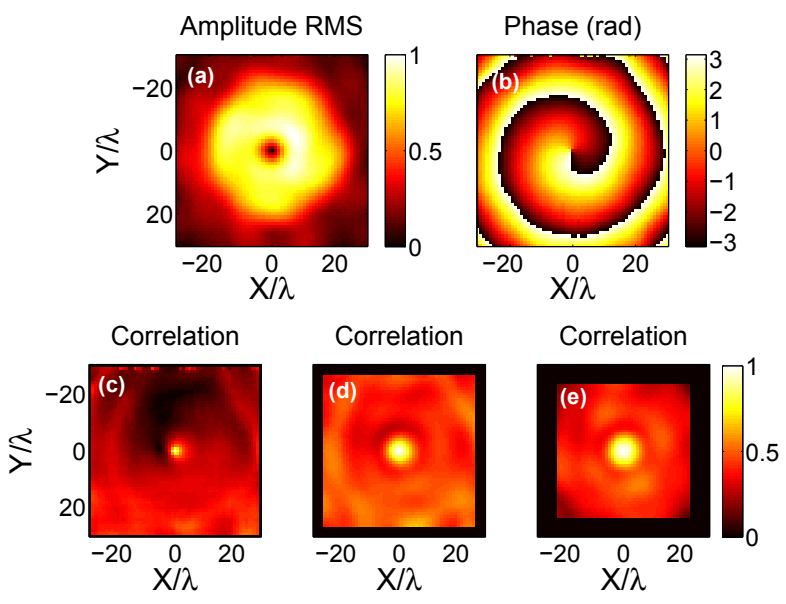

FIG. 1: (Color online) (a) Amplitude RMS and (b) phase of an experimental vortex of topological charge $l=1$. Correlation maps (see the text) obtained with different 'MatRef' sizes: (c) 3x3, (d) 9x9 and (e) 15x15 points.

In this letter, we report a shift of helical waves through off-axis circular apertures. In optics, the passage of a vortex 
core through a perfectly aligned circular aperture has been considered reporting the excitation of evanescent waves once the aperture radius is smaller than $l \lambda / 2 \pi[13]$. In acoustics, a recent study has reported that the vortex core can be moved in a controlled way by distances much shorter than the acoustic wavelength by perturbing its amplitude [14]. Here, the shift of the diffracted vortex through circular apertures is used to achieve sub-diffraction imaging of such holes with the determination of both their position and size.

An analytical form of the diffraction of a vortex can be derived. A monochromatic helical wave can be modeled as a Bessel wave [15]. In a transverse plane $(O x, O y)$ with polar coordinates $x=r \cos (\theta), y=r \sin (\theta)$, a Bessel wave $\Phi(r, \theta, z)$ is defined by:

$$
\Phi(r, \theta, z)=J_{l}(\kappa r) \exp (i l \theta) \exp \left(i k_{z} z\right) \exp (-i \omega t)
$$

with $\kappa^{2}+k_{z}^{2}=\omega^{2} / c^{2}, l \in \mathbb{Z}$ the topological charge, $\omega$ the angular frequency and $c$ the wave velocity. This model holds for either optical or acoustical vortices as long as the field can be considered scalar. We are interested in the diffraction of the vortex by a small circular aperture located close to its core and set at $z=0$. In that case, at first order in $r$, its asymptotic form is the "r-vortex" [6]:

$$
\Phi(r, \theta, z=0) \simeq \frac{1}{l !}\left(\frac{\kappa r}{2}\right)^{l} \exp (i l \theta) \exp (-i \omega t)
$$

To compute the diffracted pattern, the convenient coordinate system is centered on the circular aperture $x^{\prime}=x-x_{0}=$ $r^{\prime} \cos \left(\theta^{\prime}\right), y^{\prime}=y=r^{\prime} \sin \left(\theta^{\prime}\right)$. We choose a set of axes so that the vortex is misaligned on the $O x$ axis only, and $x_{0} \leq a$ where $a$ is the radius of the hole. When the vortex is of charge one, $l=1$, the analytical form of the vortex in the set of axes centered on the circular aperture is trivial since $r \exp (i \theta)=x+i y=x^{\prime}+x_{0}+i y=r^{\prime} \exp \left(i \theta^{\prime}\right)+x_{0}$.

$$
\Phi\left(r^{\prime}, \theta^{\prime}, z=0\right) \simeq \frac{\kappa}{2}\left(r^{\prime} \exp \left(i \theta^{\prime}\right)+x_{0}\right) \exp (-i \omega t)
$$

Consequently, the diffracted field is the superposition of the diffraction of a vortex centered on the circular aperture and a plane wave whose amplitude is proportional to the misalignment $x_{0}$. Since we are interested in the far field, the Fraunhofer diffraction will be used. With polar coordinates, the diffracted field at $z$ is :

$$
\Phi\left(r^{\prime}, \theta^{\prime}, z\right)=\frac{\kappa}{2 i \lambda z} \exp \left(i\left(k_{z} z-\omega t\right)\right) \exp \left(i \frac{k r^{\prime 2}}{2 z}\right) \int_{0}^{a} \int_{-\pi}^{\pi}\left(r^{\prime \prime} \exp \left(i \theta^{\prime \prime}\right)+x_{0}\right) \exp \left(-i \frac{k r^{\prime} r^{\prime \prime}}{z} \cos \left(\theta^{\prime}-\theta^{\prime \prime}\right)\right) d \theta^{\prime \prime} r^{\prime \prime} d r^{\prime \prime}
$$

Using the integral definition of the Bessel functions, the last equation can be rewritten:

$$
\Phi\left(r^{\prime}, \theta^{\prime}, z\right)=\frac{\pi \kappa}{i \lambda z} \exp \left(i\left(k_{z} z-\omega t\right)\right) \exp \left(i \frac{k r^{\prime 2}}{2 z}\right) \int_{0}^{a}\left(r^{\prime \prime} \exp \left(i\left(\theta^{\prime}-\pi / 2\right)\right) J_{1}\left(\frac{k r^{\prime} r^{\prime \prime}}{z}\right)+x_{0} J_{0}\left(\frac{k r^{\prime} r^{\prime \prime}}{z}\right)\right) r^{\prime \prime} d r^{\prime \prime}
$$

We are interested in the far field a few wavelengths away from the axis $r^{\prime} / z<<1 \Leftrightarrow k r^{\prime} r^{\prime \prime} / z<<1$ so we can use the same first order approximation of the Bessel function around the origin as above to get:

$$
\Phi\left(r^{\prime}, \theta^{\prime}, z\right)=\frac{\pi \kappa}{i \lambda z} \exp \left(i\left(k_{z} z-\omega t\right)\right) \exp \left(i \frac{k r^{\prime 2}}{2 z}\right) \int_{0}^{a}\left(\frac{k r^{\prime}}{2 z} \exp \left(i\left(\theta^{\prime}-\pi / 2\right)\right) r^{\prime \prime 3}+x_{0} r^{\prime \prime}\right) d r^{\prime \prime}
$$

That expression can be easily integrated

$$
\Phi\left(r^{\prime}, \theta^{\prime}, z\right)=-\frac{\pi^{2} \kappa a^{4}}{4 \lambda^{2} z^{2}} \exp \left(i \frac{k r^{\prime 2}}{2 z}\right)\left(x^{\prime}+i\left(y^{\prime}+\frac{2 z x_{0} \lambda}{\pi a^{2}}\right)\right) \exp \left(i\left(k_{z} z-\omega t\right)\right)
$$

Thus, the vortex shift is perpendicular to the displacement of the circular aperture. It is proportional to the distance $z$ so we can introduce an angle of deviation: $\arctan \left(\frac{2 x_{0} \lambda}{\pi a^{2}}\right)$. The shift of the diffracted vortex is proportional to the original shift $x_{0}$ and inversely proportional to the surface of the aperture. The existence of a transverse momentum for an optical vortex scattered by an off-axis aperture was inferred in relation with the intrinsic and extrinsic nature of the orbital angular momentum [16]. However the transverse shift of the scattered vortex whose rotation axis remains directed on the $O z$ axis is remarkable and provides a mean to measure the radius of the aperture. The scattered amplitude decreases as the fourth power of the radius of the aperture whereas the Rayleigh scattering is only quadratic with the amplitude. This difference comes from the linear radial dependence of the incident vortex and also from the azimuthal dependence that leads to a Bessel function of first order, Eq. 5. This is obviously the most severe drawback of this technique. 
Here, we propose a correlation method in order to detect and localize precisely the phase singularity $\left(x_{\text {sing }}, y_{\text {sing }}\right)$; thus measuring the shift of the diffracted vortex. Let us consider, for instance, a vortex scanned on a grid made of 61x61 points as shown on Figs. 1(a,b). First, we extract a set of points centered on the phase singularity. That defines several 'Matrices of Reference' - denoted 'MatRef' in the following paragraphs - of different sizes $N \times N$. For each of them, the correlation function with the original measurements made on the whole grid of 61x61 is computed (Figs. $1(\mathrm{c}, \mathrm{d}, \mathrm{e}))$. Obviously, the correlation is excellent at the center because the vortex core includes the phase singularity, whose position is given by the maximum of the correlation coefficient $(=1)$. On the other hand, the correlation coefficient decreases by moving away from the center. In addition, by comparing Figs. 1(c,d,e), one can note that the smaller the 'MatRef' size is, the sharper the correlation is. Thus, if we neglect the experimental limitations - e.g. the signal-to-noise ratio in the vortex core - the accuracy of this technique only depends on the 'MatRef' size. The result is a non-classical resolution which is nonexistent with other classical waves.

To illustrate the previous analysis, we perform acoustic experiments underwater by using a recently developed setup [17]. That latter is made of a spherically focused array of 127 piezoelectric transducers mounted on a spherical cap with a geometrical focal length $F=450 \mathrm{~mm}$ and a diameter aperture $D=100 \mathrm{~mm}$, immersed in water as shown in Fig. 2. The origin of the $O z$ axis is fixed at the focal point, so that the array of transducers is located in the plane $z=-450$ $\mathrm{mm}$. The central frequency of the transducers is $1 \mathrm{MHz}$ and they are excited by wave trains centered at the same frequency corresponding to a time period $T=1 \mu \mathrm{s}$ and a wavelength $\lambda=1.5 \mathrm{~mm}$. Then, we use the linear inverse filter technique [18] to synthesize acoustical vortices with a width equal to $10 \lambda$ in the focal plane $z=0 \mathrm{~mm}$.

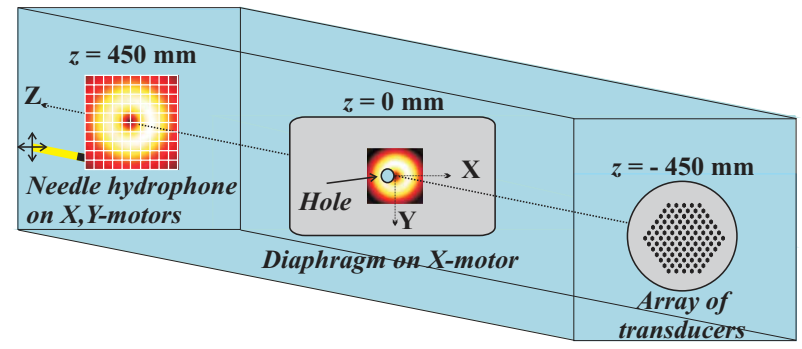

FIG. 2: (Color online) Experimental setup immersed in water.

To simulate a circular aperture, we insert an 'acoustic diaphragm' made of stainless steel in the focal plane. This high acoustic impedance plate is much larger than the propagating vortex extension. We use various perforated plates with holes of diameters $d=\lambda, 2 \lambda$ and $4 \lambda$. In comparison, the limited resolution due to diffraction is equal to $\lambda F / D=4.5 \lambda$ in our experiments. The diaphragms are mounted on a stepper motor to control the lateral displacements along the $O x$ axis. Finally, we align the array of transducers with the hole as accurate as possible. Then, we measure the instantaneous amplitude of the acoustic field diffracted by the circular aperture in the plane $z=450 \mathrm{~mm}$, thanks to a needle hydrophone of $1 \mathrm{~mm}$ in diameter (Precision Acoustics Ltd, UK). The recorded signals are averaged over 32 times in order to improve the signal-to-noise ratio. The needle hydrophone is moved by stepper motors in both transverse directions $(O x, O y)$. Thus, pressure can be recorded on a surface as shown on Figs. 1(a,b) for classical features of a vortex.

Then, once the vortex position is approximately localized, its core - i.e. the zero-amplitude area - is scanned more precisely with $61 \times 61$ points regularly set on a square grid of $30 x 30 \mathrm{~mm}^{2}$ with a spatial step of $\lambda / 3=0.5 \mathrm{~mm}$. A more accurate scan would not provide us with more information because of the spatial averaging on the hydrophone surface $\left(\sim 1 \mathrm{~mm}^{2}\right)$. So, we interpolate the measured acoustic field numerically by 20 to obtain a finer meshing.

Let us consider the most interesting case, namely the smallest hole of diameter $d=\lambda$. Fig. 3 shows that, while the diaphragm is moved along the $O x$ axis, the phase singularity also shifts along the same transverse direction. As previously explained, the correlation map displays the precise position of the phase singularity. Thus, we are able to measure precisely its lateral displacement $x_{s i n g}$ as a function of the lateral displacement of the circular aperture $x_{0}$. Figs. 4(a,b,c) show the measurements of $x_{\text {sing }}$ in function of $x_{0}$ for the various diameters, respectively $d=\lambda, d=2 \lambda$ and $d=4 \lambda$. The figures reveal a linear dependence between these two parameters for each diameter. Then, linear regressions are performed and the fitting parameters are given in the insets of Figs. 4(a,b,c). Fitting parameter 'b' corresponds to the 'shift velocity' of the phase singularity. Fitting parameter 'a' corresponds to its position along the $O x$ axis when the circular aperture is perfectly centered in the plane $\mathrm{z}=0 \mathrm{~mm}$. Let us note that the fitting parameter 'a' is not equal to 0 in our experiments, as shown in the insets of Figs. $4(\mathrm{a}, \mathrm{b}, \mathrm{c})$. This is probably due to a slight misalignment.

Contrary to the optical domain, perfectly opaque diaphragms do not exist in acoustics. Therefore stainless steel 

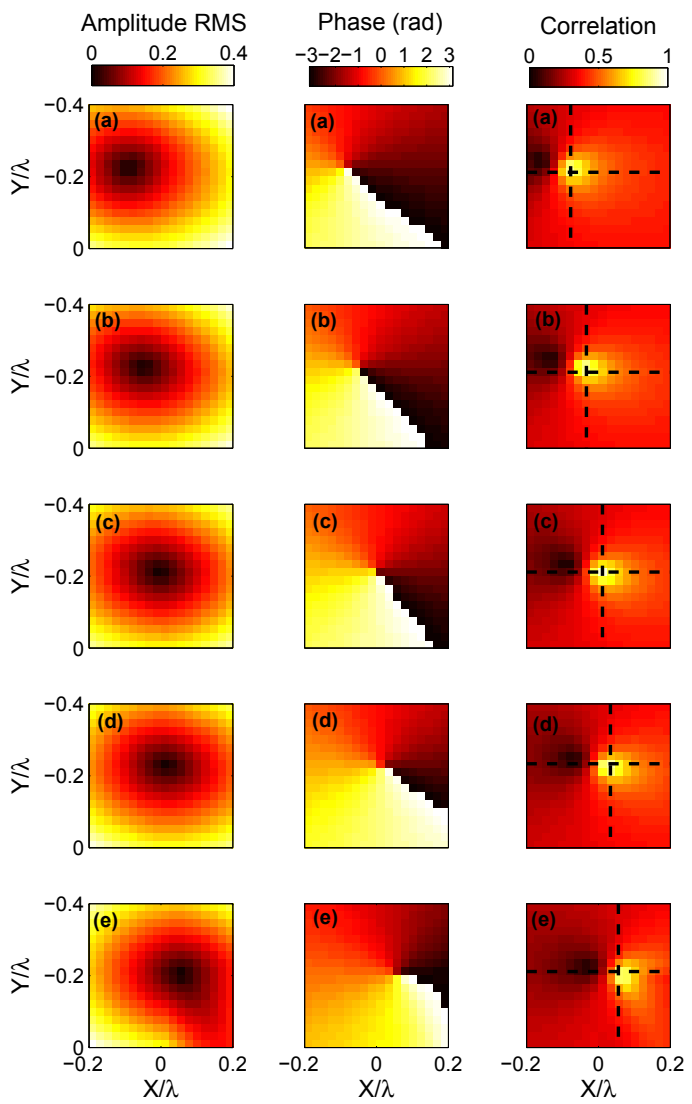

FIG. 3: (Color online) Singularity shift observed in the plane $z=450 \mathrm{~mm}$ while diaphragm shifts: (a) $x_{0}=-\lambda / 2,(\mathrm{~b}) x_{0}=-\lambda / 4$, (c) $x_{0}=0$, (d) $x_{0}=\lambda / 4$, (e) $x_{0}=\lambda / 2$. From left to right: amplitude RMS, phase and correlation coefficients. Dash lines indicate the position of the detected phase singularity $\left(x_{\text {sing }}, y_{\text {sing }}\right)$ thanks to the correlation method (see the text).
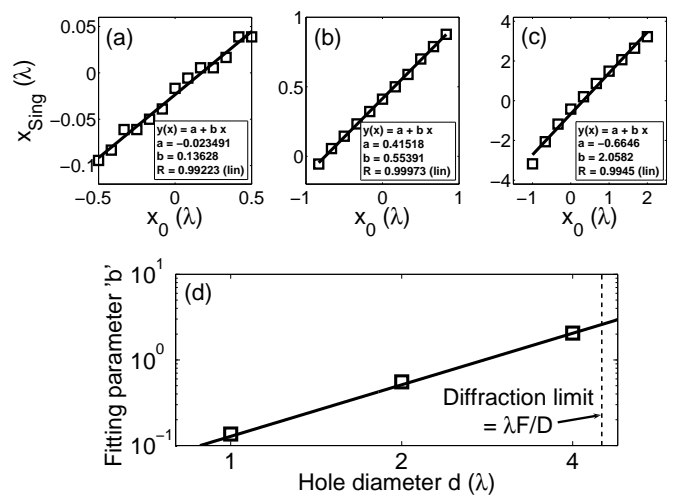

FIG. 4: Singularity shift $\left(x_{\text {sing }}\right)$ VS diaphragm shift $\left(x_{0}\right)$ for three hole diameters: (a) $d=\lambda$, (b) $d=2 \lambda$ and (c) $d=4 \lambda$. Insets correspond to the linear fit values of experimental data. (d) Fitting parameter $b$ (in log-log scale): experimental data ( $\square$ ) and theoretical predictions (solid curve, see the text). The vertical dash line refers to the diffraction limit.

plates used here can only be considered as 'semi-opaque'. To reduce the transmission even more, we chose a $\lambda_{\text {steel }} / 4$ thickness. But even in that case the transmitted wave can not be neglected. Therefore, we get the superposition of a transmitted vortex centered at $(x=0, y=0)$ and a scattered vortex located at $\left(x=x_{0}, y=-\frac{4 z x_{0}}{k a^{2}}\right)$. The amplitude of the scattered vortex is $S=-\frac{\pi^{2} a^{4}}{4 \lambda^{2} z^{2}} \exp \left(i \frac{k r^{2}}{2 z}\right)$ whereas for the transmitted one, we must take into account: the complex transmission factor of the stainless steel plate, $T$, and the diffraction from the circular aperture to the plane of measurement. The last effect results in a complex coefficient due to the spreading of energy and the Gouy phase. 
This coefficient was measured by numerically back-propagating the field measured at $z=450 \mathrm{~mm}$ in free space. The result is $\exp (i \pi) / 6.56$ with the approximation of Eq. 2. The resulting total field is:

$$
\Phi^{T}\left(r^{\prime}, \theta^{\prime}, z\right)=\kappa\left(-\frac{T}{6.5}+S\right)\left(x-x_{0}\left[\Re(\alpha)-\frac{2 z \lambda}{\pi a^{2}} \Im(\alpha)\right]+i\left\{y-x_{0}\left[\Im(\alpha)+\frac{2 z \lambda}{\pi a^{2}} \Re(\alpha)\right]\right\}\right)
$$

with $\alpha=S /(-T / 6.5+S)$. The factor of proportionality now depends on both the aperture radius and the stainless steel plate transmission factor. However the scattered field is so weak that we may even consider that $|T|>>|S|$. The phase shift due to the thickness of the plate is $-\left(\omega / c_{\text {steel }}\right)\left(\lambda_{\text {steel }} / 4\right)\left(1-c_{\text {steel }} / c_{\text {water }}\right)=-\pi / 2(1-5.8 / 1.48)=2.92 \pi / 2$ and we can get the factor $\exp (i 2.92 \pi / 2) \simeq-0.13-0.99 i$. Note that close to the axis $S$ is purely real. Thus, we use the approximation: $\alpha \simeq-6.5(0.13+0.99 i) S /|T|$. The previous expression can be simplified to:

$$
\Phi^{T}\left(r^{\prime}, \theta^{\prime}, z\right) \simeq-\left(\frac{\kappa T}{6.5}\right)\left(x-x_{0} \frac{12.9 z \lambda}{\pi a^{2}} \frac{S}{|T|}+i\left\{y+x_{0} \frac{1.6 z \lambda}{\pi a^{2}} \frac{S}{|T|}\right\}\right)
$$

The phase shift due to the thickness of the stainless steel plate leads to a scattered vortex shifted on the $O x$ axis. The shift is proportional to $x_{0}$ with a coefficient of proportionality now equal to $\left(12.9 z \lambda / \pi a^{2}\right)(S /|T|)$ that behaves as $1 / a^{2}$. The slope $b$ can now be computed and is equal to $(12.9 \pi \lambda / 16 z)(d / \lambda)^{2}(1 / T)$. The transmission factor can also be derived by computing the geometric series describing the multiple reflections inside the stainless steel plate. The result is $T=2 Z_{\text {water }} / Z_{\text {steel }} \simeq 1 / 15.3$, where $Z$ is the impedance, that has been checked experimentally. The final result is $b=0.129(d / \lambda)^{2}$ and is reported in Fig. 4(d). It shows an excellent quantitative agreement between the experiments $(\square)$ and the theoretical analysis (solid curve).

Using singular waves, the size and the location of a hole about 5 times smaller than the classical limit of resolution has been recovered. This demonstrates that singular waves can be used to perform sub-diffraction imaging in acoustics as well as in optics as long as scalar fields are considered. To achieve this goal, we have detected the location of the phase singularity once the vortex has been diffracted by the circular aperture thanks to a correlation with a vortex of reference exploiting the super-oscillation property. However, unexpectedly, an incident beam with a non-zero topological charge is diffracted sideways and the amount of deviation is proportional to the misalignment and the inverse of the surface of the hole. Therefore, this technique is not limited by the size of the hole. The main limitations are: on the one hand, the poor signal-to-noise ratio since the amplitude of the helical beam tends towards zero as the super-oscillation tends toward infinity close to the vortex axis. On the other hand, the dynamic range required to image more complex objects is very large since the amplitude of the wave scattered by an on-axis hole varies as the square of Rayleigh scattering amplitude. In a future work, we will study smaller objects - e.g. about a fraction of the wavelength - in order to achieve sub-wavelength imaging.

* Electronic address: jean-louis.thomas@upmc.fr

[1] Z. Liu, H. Lee, Y. Xiong, C. Sun, and X. Zhang, Science 315, 1686 (2007)

[2] J. Li, L. Fox, X. Yin, G. Bartal, and X. Zhang, Nature Mater. 8, 931 (2009)

[3] N. I. Zheludev, Nature Mater. 7, 420 (2008)

[4] M. V. Berry and S. Popescu, J. Phys. A: Math. Gen. 39, 69656977, (2006)

[5] M. V. Berry, Waves near zeros Coherence and Quantum Optics IX (Optical Society of America, Washington DC, 2008) $37(2008)$

[6] J. F. Nye and M. V. Berry, Proc. R. Soc. London Ser. A 336, 165 (1974)

[7] M. S. Soskin and M. V. Vasnetsov, Prog. Opt. 42221 (2001)

[8] L. Allen, M. W. Beijersbergen, R. J. C. Spreeuw, and J. P. Woerdman, Phys. Rev. A 458185 (1992)

[9] N. B. Simpson, K. Dholakia, L. Allen, and M. J. Padgett, Opt. Lett. 22, 52 (1997)

[10] B. T. Hefner and P. L. Marston, J. Acoust. Soc. Am. 106, 3313 (1999)

[11] J.-L. Thomas and R. Marchiano, Phys. Rev. Lett. 91, 244302 (2003)

[12] K. Volke-Sepúlveda, A. O. Santillań, and R. R. Boullosa, Phys. Rev. Lett. 100, 024302 (2008)

[13] R. Zambrini, L.C. Thomson, S.M. Barnett, and M.J. Padgett, J. Mod. Opt. 52, 1135 (2005)

[14] C. Wilson and M.J. Padgett, New J. Phys. 12, 023018 (2010)

[15] J.-L. Thomas, T. Brunet, and F. Coulouvrat, Phys. Rev. E 81, 016601 (2010)

[16] A.T. O'Neil, I. MacVicar, L. Allen, and M. J. Padgett, Phys. Rev. Lett. 88053601 (2002)

[17] T. Brunet, J.-L. Thomas, R. Marchiano, and F. Coulouvrat, New J. Phys. 11, 013002 (2009)

[18] M. Tanter, J.-L. Thomas, and M. Fink, J. Acoust. Soc. Am. 108, 223 (2000) 